\title{
Desenvolvimento de protocolos de dosimetria interna empregando o código MCNPx e fantomas voxelizados de referência da ICRP 110
}

\author{
B. M. Mendes ${ }^{\mathrm{a}, \mathrm{b}}$; T. C. F. Fonseca ${ }^{\mathrm{a}}$; B. M. Trindade ${ }^{\mathrm{b}}$; T. P. R. Campos ${ }^{\mathrm{b}}$ \\ ${ }^{a}$ Seção de Dosimetria das Radiaçães - SECDOS, Centro de Desenvolvimento da Tecnologia Nuclear - CDTN/CNEN, \\ 30.161-970, Belo Horizonte - MG, Brasil. \\ ${ }^{b}$ Departamento de Engenharia Nuclear - DEN, Universidade Federal de Minas Gerais - UFMG,
} 31270-901 Belo Horizonte - MG, Brasil

bmm@cdtn.br

\begin{abstract}
RESUMO
O objetivo desse trabalho foi realizar cálculos de dosimetria interna para ${ }^{18} \mathrm{~F}-\mathrm{FDG}$ empregando o código MCNPx e os fantomas de referência voxelizados da ICRP 110 (RCP_AF e RCP_AM). As metodologias desenvolvidas e validadas nesse estudo representam protocolos de dosimetria interna contendo uma melhor representação antropométrica e antropomórfica do modelo humano onde distribuições heterogêneas das emissões podem ser adotadas, uteis no estudo de novos radiofármacos e casos de contaminação interna. Os fantomas de referência foram implementados para rodar no MCNPx. Dados de biodistribuição referentes ao radiofármaco ${ }^{18} \mathrm{~F}-\mathrm{FDG}$ fornecidos na ICRP 128 foram utilizados nas simulações. As doses absorvidas médias nos órgãos e a dose efetiva foram calculadas para cada modelo. Os valores obtidos foram comparados com dois trabalhos de referência disponíveis na literatura para fins de validação. As médias das diferenças entre os valores calculados nesse estudo e os valores de referência de Zankl et al., 2012 foram -0,3\% para RCP_AM e -0,4\% para RCP_AF. Considerando os valores de referência de Hadid et al., 2013 as médias dos desvios foram $-2,9 \%$ e $-2,2 \%$ para RCP_AM e RCP_AF respectivamente. Não foram observadas diferenças estatisticamente significantes $(\mathrm{p}<0,01)$ entre os valores de referência e os valores calculados por meio dos protocolos de dosimetria interna desenvolvidos por nosso grupo. Considerando a validação realizada nesse estudo para administração intravenosa de ${ }^{18} \mathrm{~F}-\mathrm{FDG}$, os protocolos de dosimetria interna desenvolvidos reproduzem dados dosimétricos considerados satisfatórios.
\end{abstract}




\begin{abstract}
The objective of this work was to perform internal dosimetry calculations for ${ }^{18} \mathrm{~F}-\mathrm{FDG}$ employing the MCNPx code and ICRP 110 voxelized reference phantoms (RCP_AF and RCP_AM). The methodologies developed and validated here represent protocols of internal dosimetry holding a better anthropomorphic and anthropometric representation of the human model in which heterogeneous distributions of the emissions can be adopted, useful in the study of new radiopharmaceuticals and internal contamination cases. The reference phantoms were implemented to run on MCNPx. Biodistribution data of ${ }^{18} \mathrm{~F}$-FDG radiopharmaceutical provided in ICRP 128 were used in the simulations. The organs average absorbed doses and the effective doses were calculated for each model. The values obtained were compared with two reference works available in the literature for validation purposes. The means of the difference of our values and Zankl et al., 2012 reference values were $-0.3 \%$ for RCP_AM and $-0.4 \%$ for RCP_AF. Considering Hadid et al., 2013 reference values, the means of the deviation were $-2.9 \%$ and $-2.2 \%$ for $\mathrm{RCP} \_\mathrm{AM}$ and $\mathrm{RCP}$ AF respectively. No statistically significant differences were observed $(\mathrm{p}<0.01)$ between the reference values and the values calculated by the internal dosimetry protocols developed by our group. Considering the ${ }^{18}$ F-FDG validation study performed in this work, the internal dosimetry protocols developed by our group have produced suitable dosimetry data.
\end{abstract}

Keywords: Internal Dosimetry, MCNPx, Validation. 


\section{INTRODUÇÃO}

A Comissão Internacional de Proteção Radiológica (ICRP) e a Comissão Internacional de Unidades em Radiação (ICRU) disponibilizaram em 2009 os fantomas voxelizados realísticos de referência do homem e da mulher adultos [1]. Tais fantomas, denominados RCP_AM e RCP_AF, substituíram os modelos analíticos estilizados na geração e avaliação de dados de referência em cálculos dosimétricos envolvendo radiações ionizantes [1].

Nosso grupo tem trabalhado no desenvolvimento de modelos e protocolos para cálculos computacionais especialmente para dosimetria interna de radiofármacos e de situações de contaminação radioativa [4, 5, 6, 7]. A validação desses modelos e protocolos de cálculo é fundamental para garantir a confiabilidade dos resultados gerados.

Uma forma usual de validação de cálculos dosimétricos computacionais é a comparação dos valores obtidos nas simulações com valores de trabalhos de referência disponibilizados na literatura. Zankl et al. [8] utilizaram os fantomas RCP_AM e RCP_AF e obtiveram dados de dose absorvida nos órgãos e dose efetiva por atividade administrada para ${ }^{18} \mathrm{~F}-\mathrm{FDG},{ }^{123} \mathrm{I}$-BMIPP e ${ }^{68} \mathrm{Ga}$-EDTA. Hadid et al. [9] também utilizaram os mesmos fantomas para calcular dose absorvida nos órgãos e dose efetiva para diversos radiofármacos, incluindo ${ }^{18} \mathrm{~F}$-FDG.

O objetivo desse estudo foi realizar cálculos de dosimetria interna para ${ }^{18} \mathrm{~F}$-FDG utilizando o código MCNPx e os fantomas de referência da ICRP [1]. As metodologias desenvolvidas e validadas nesse estudo representam protocolos de dosimetria interna contendo uma melhor representação antropométrica e antropomórfica do modelo humano onde distribuições heterogêneas das emissões podem ser adotadas, uteis em estudos de novos radiofármacos e casos de contaminação interna. Tais protocolos serão de grande importância em estudos específicos, envolvendo uma dosimetria personalizada, baseada em imagens anatômicas e moleculares corregistradas de pacientes. Além disso, espera-se que as descrições metodológicas venham auxiliar pesquisadores que desejam iniciar seus trabalhos no campo da dosimetria interna.

Os dados de dose absorvida por órgão e dose efetiva para ${ }^{18}$ F-FDG apresentados nos trabalhos de Zankl et al. [8] e Hadid et al. [9] foram considerados Valores de Referência (VR) para efeito de validação. 


\section{MATERIALS AND METHODS}

\subsection{Preparação dos modelos representativos humanos masculino e feminino.}

Dois arquivos de entrada foram gerados para o MCNPx versão 2.7.0 [10], representando modelos tridimensionais de voxels relativos aos fantomas RCP_AM e RCP_AF. Para converter os dados em formato '. dat' ao formato de entrada estabelecido no MCNPx foi desenvolvido um programa in house. As composições atômicas e densidades de cada material associado a um tecido foram obtidas na ICRP 110 [1]. O cartão MODE foi implementado como "MODE p e" para habilitar o transporte de fótons e elétrons. O cartão de fonte (SDEF) foi implementado com emissões de pósitrons (PAR= -3) do ${ }^{18} \mathrm{~F}$. O transporte da energia dos pósitrons também foi acompanhado até a aniquilação da partícula. O espectro de energia dos pósitrons foi obtido na ICRP 107 [11]. A probabilidade de emissão nos voxels de determinado tecido foi baseada nos dados biocinéticos para ${ }^{18} \mathrm{~F}-\mathrm{FDG}$ constantes na ICRP 128 [12]. De acordo com as recomendações da publicação ICRP 133, regiõesfonte constituídas por um único tecido apresentaram probabilidade de emissão uniforme de pósitrons. Algoritmos de amostragem foram utilizados para as regiões fonte-contendo constituídas por dois ou mais tecidos diferentes, levando em consideração a massa de cada tecido [3].

Os órgãos-fonte são: Bexiga (conteúdo), Cérebro, Coração (parede), Fígado, Pulmão (tec. + sangue), Outros Órgãos e Tecidos. A expressão "outros órgãos e tecidos" refere-se todos os tecidos do fantoma exceto os tecidos com fonte distribuída interna, ditos tecidos-fontes e os tecidos-nãoemissores. Os tecidos-não-emissores incluem os conteúdos de órgãos com parede definida (vesícula biliar, estomago, intestino delgado e cólons ascendente transverso descendente e sigmoide) e os voxels de ar dentro e fora do corpo.

O tally +F6 foi empregado para solicitar a dose média (MeV/g) nos órgãos e tecidos do modelo. Tanto fótons como elétrons são considerados no cômputo das doses nesse tipo de pedido de saída. O transporte de partículas secundárias é levado em consideração. O algoritmo de indexação de energia ITS foi habilitado (DBCN 17j 1). Tal algoritmo considerado mais acurado que a opção “defaut" quando se utiliza o MCNPx para o transporte de elétrons [13]. A energia de "cutoff” foi de $1 \mathrm{KeV}$ para fótons, elétrons e pósitrons. O número de histórias acompanhadas em cada simulação (NPS) foi de $2 \mathrm{E}+7$. 


\subsection{Processamento dos dados simulados}

O conjunto de valores de dose absorvida média por partícula emitida na fonte $\left[\mathrm{D}_{\text {tec }}\right.$, em unidade $\mathrm{MeV} \cdot \mathrm{g}^{-1} \cdot \mathrm{p}^{-1}$ ] e erro relativo [ER s/un.] para cada tecido do modelo são lidos nos arquivos de saída oriundos das simulações, como a média dos valores dos voxels contidos pelo tecido. A sigla "p" na unidade dos valores gerados no MCNP representa partícula emitida na simulação. Os valores, em $\mathrm{MeV} \cdot \mathrm{g}^{-1} \cdot \mathrm{p}^{-1}$, são convertidos em Gy. $\mathrm{p}^{-1}$. O desvio padrão (SD) foi calculado considerando o erro relativo, tal que $\mathrm{SD}=\mathrm{ER} * \mathrm{D}_{\text {tec}}$. Um arquivo “.csv" contendo a dose absorvida média por unidade de emissão $\left(\mathrm{Gy} \cdot \mathrm{p}^{-1}\right)$, o erro relativo (\%) e o desvio padrão $\left(\mathrm{Gy} \cdot \mathrm{p}^{-1}\right)$ para cada tecido do modelo foi gerado. A partir dos valores de dose absorvida média por partícula emitida foi elaborado o cálculo da dose no órgão por atividade injetada $\left(\mathrm{mGy} \cdot \mathrm{MBq}^{-1}\right)$.

A dose no órgão por atividade injetada $\left(\mathrm{mGy} \cdot \mathrm{MBq}^{-1}\right), \mathrm{D}_{\text {org }}$, para fluor-18 foi avaliado como segue:

$$
\mathrm{D}_{\text {org }}=\mathrm{D}_{\mathrm{tec}} \cdot \mathrm{P}_{\mathrm{dec}} \cdot \tau_{\mathrm{FDG}} \cdot \mathrm{FC}
$$

Para órgãos constituídos por apenas um tecido, como o fígado, $\mathrm{D}_{\text {tec }}$ é a dose absorvida média no tecido que compõe o órgão por unidade de emissão $\left(\mathrm{Gy} \cdot p^{-1}\right)$; enquanto, para órgãos constituídos por mais de um tecido foi considerada a média entre as doses de cada tecido que o compõe ponderada por suas respectivas massas. $\mathrm{O}$ valor de $\mathrm{D}_{\text {tec }}$ é calculado pelo MCNPx em $\left(\mathrm{MeV} \cdot \mathrm{g}^{-1} \cdot p^{-1}\right)$ e convertido para $\left(\mathrm{Gy} . \mathrm{p}^{-1}\right)$ multiplicando-se o valor em MeV.g $\mathrm{g}^{-1}$ por 1,602E-10. $\mathrm{P}_{\text {dec }}$ é o número de pósitrons por transformação, $t$ (decaimento) do flúor-18 $\left(0,9673 p . t^{-1}\right)$ fornecido pelo software DECDATA da ICRP 107 [11]. Por sua vez, $\tau_{\mathrm{FDG}}$ é o tempo total de residência do ${ }^{18} \mathrm{~F}$-FDG (8960,4 s) obtido na ICRP 128 [12]. FC é o fator de conversão de Gy.Bq ${ }^{-1}$ para $\mathrm{mGy}_{\mathrm{MBq}} \mathrm{MB}^{-1}(\mathrm{FC}=1 \mathrm{E}+09)$.

Metodologias especiais foram adotadas para o cálculo da dose absorvida média na medula óssea vermelha (MOV), endósteo e colón de acordo com os trabalhos de Zankl et al. [8] e Hadid et al. [9].

A dose efetiva foi calculada pelo programa a partir das doses absorvidas nos órgãos obtidas na etapa anterior. Os fatores de peso por tecido $\left(\mathrm{w}_{\mathrm{t}}\right)$ e pelo tipo de radiação $\left(\mathrm{w}_{\mathrm{r}}\right)$ foram obtidos na ICRP $103[2]$. 


\subsection{Validação}

Os valores de dose absorvida média por atividade administrada (mGy. $\mathrm{MBq}^{-1}$ ) para cada órgão e dose efetiva por atividade administrada $\left(\mathrm{mSv} \cdot \mathrm{MBq}^{-1}\right)$ obtidos nas simulações realizadas para os fantomas RCP_AM e RCP_AF foram comparados com os apresentados na literatura [8, 9]. Testes de hipótese foram realizados para determinar se as diferenças encontradas poderiam ser consideradas estatisticamente significantes. Tendo em vista que os trabalhos de referência não apresentaram erros relativos específicos para a dose absorvida em cada órgão (apenas menciona-se em geral que as incertezas estatísticas foram menores que 5\%) o erro relativo dos valores apresentados foi definido em 5\%. O nível de significância adotado nos testes de hipótese foi de 0,01 .

\section{RESULTADOS}

Os protocolos de dosimetria interna (PDI) desenvolvidos estão ilustrados na figura 1, onde os processos e fluxos de informação foram descritos. A etapa de preparação dos arquivos de entrada destaca-se em verde. A cor azul evidencia a fase de processamento dos dados simulados.

O número de histórias acompanhadas (NPS) de 2E7 foi suficiente para manter os erros relativos abaixo de $5 \%$ em todos os tecidos segmentados no fantoma masculino e no feminino. As únicas exceções foram os cristalinos (eye lens) que devido ao seu pequeno volume tiveram ER superiores a $5 \%$, mas inferiores a $10 \%$.

As tabelas 1 e 2 exibem os valores de dose absorvida média nos órgãos dos RCP_AM e RCP_AF e dose efetiva por unidade de atividade de ${ }^{18}$ F-FDG injetada, calculados por meio dos Protocolos de Dosimetria Interna (PDI) desenvolvidos por nosso grupo. A aplicação do teste de hipóteses, considerando o nível de significância de 0,01 e o erro relativo de 5\% para os VR, demonstrou que os valores obtidos nesse trabalho não foram diferentes dos valores de referência considerando o nível de significância ( $\mathrm{p}<0,01$ ), ou seja, todos os valores-p foram maiores que 0,01. Da mesma forma, não foram observadas diferenças estatisticamente significativas entre os dois conjuntos de valores de referência. 
Figura 1: Fluxograma dos PDI desenvolvidos. Destacam-se as etapas de preparação dos arquivos de entrada (verde) e de processamento dos dados simulados (azul)

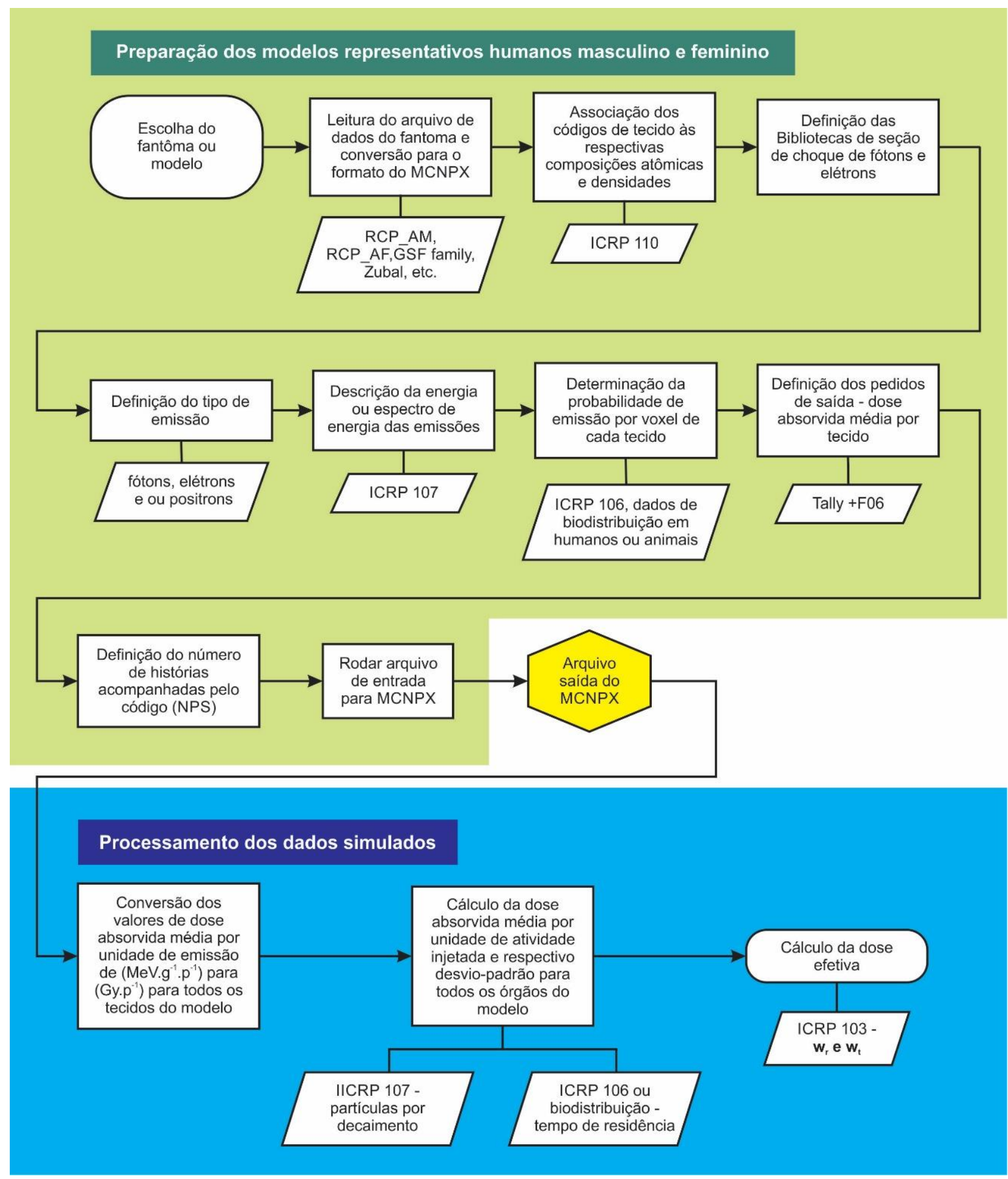




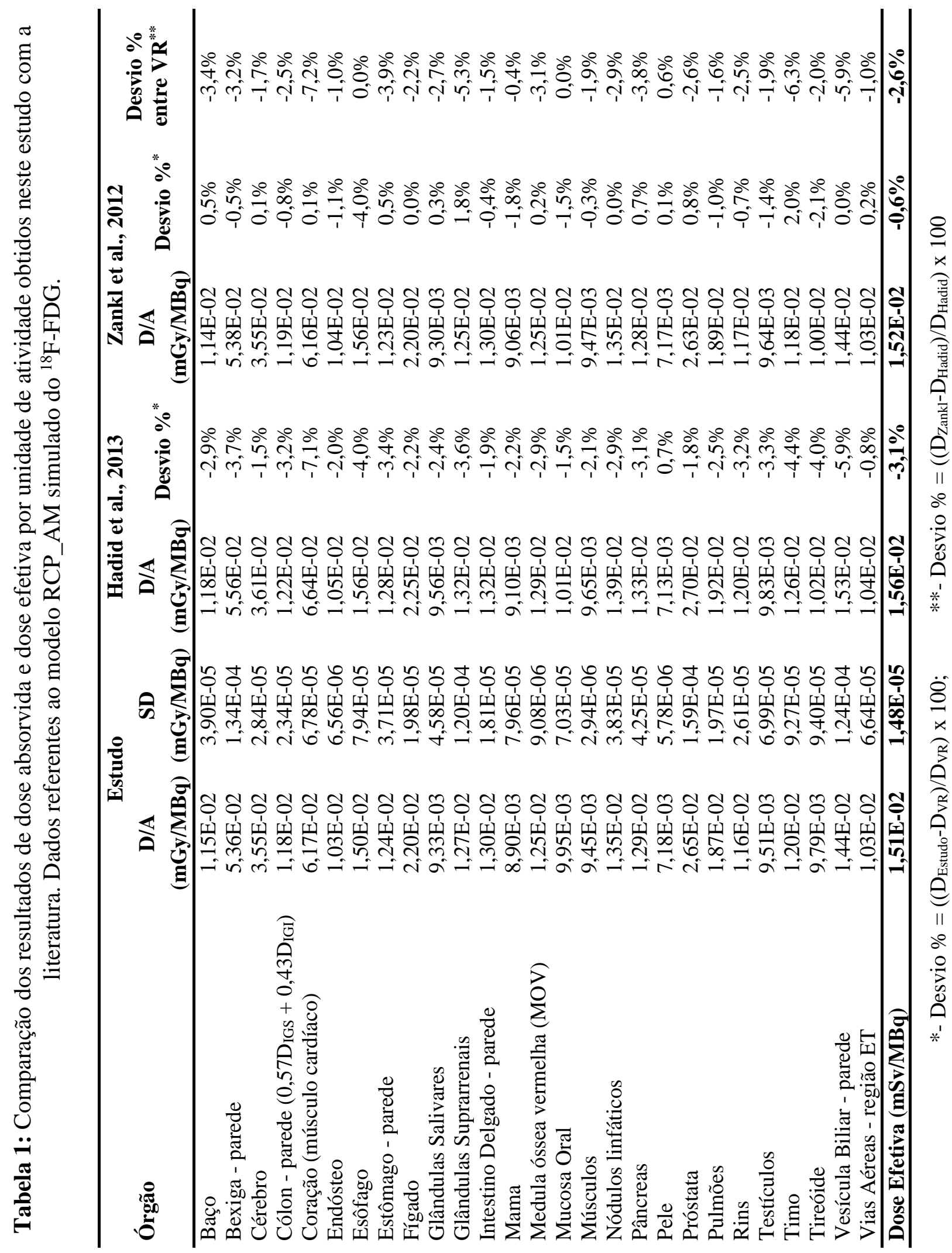




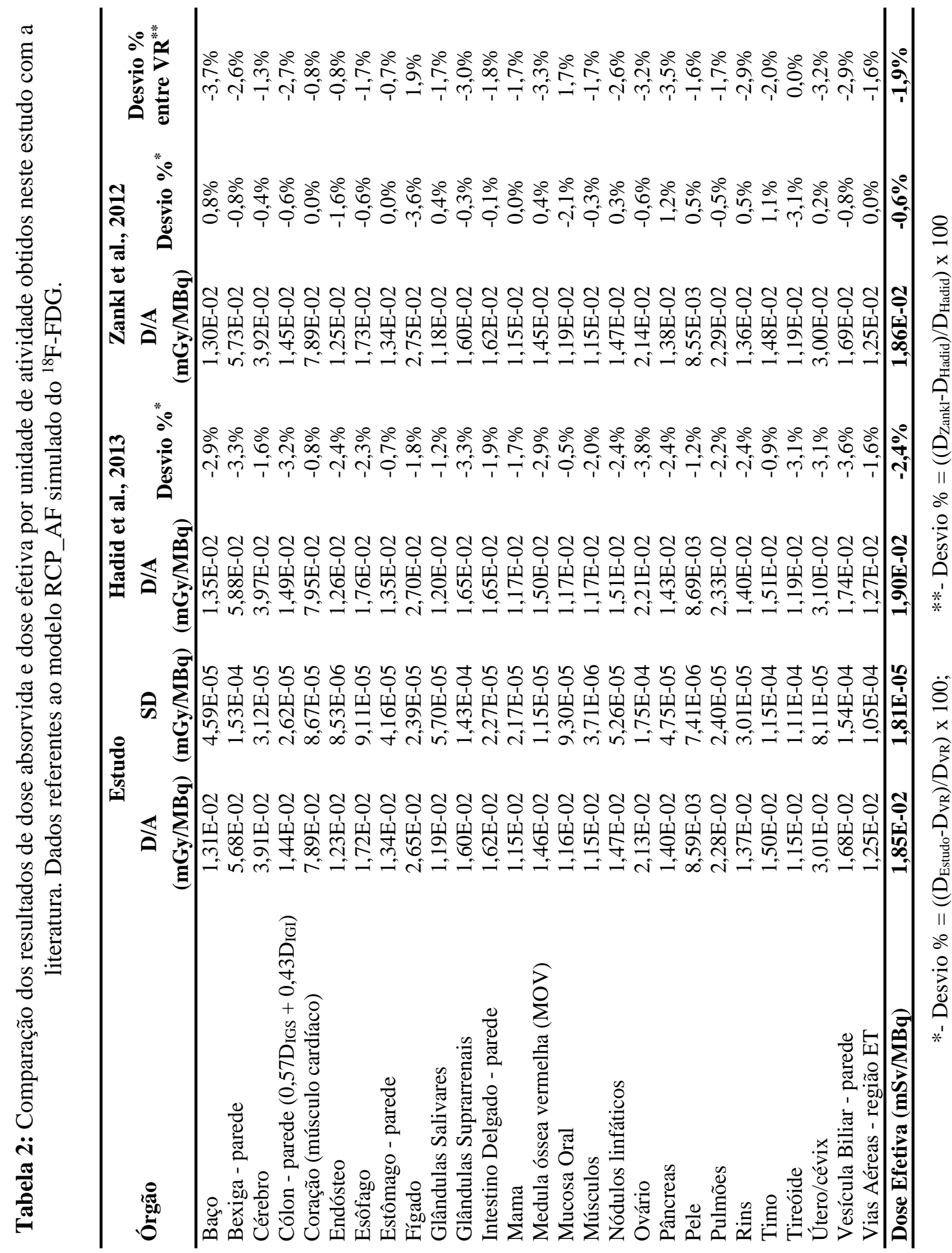


Considerando o fantoma RCP_AM, os valores calculados por meio dos PDI desenvolvidos apresentaram maior concordância com os VR de Zankl et al. [8]. O desvio em relação à referência foi menor que 5\% para todos os órgãos. Os valores calculados nesse estudo, apesar de não serem significativamente distintos dos resultados de Hadid et al. [9], foram sistematicamente menores. As maiores diferenças foram: músculo cardíaco $(-7,1 \%)$ e parede da vesícula biliar $(-5,9 \%)$. As diferenças entre as doses efetivas foram menores que 3,2\% para RCP_AM.

Quanto ao fantoma feminino (RCP_AF), os valores publicados por Zankl et al. [8] também apresentaram melhor correlação com os valores obtidos nesse trabalho. Os desvios ficaram abaixo de $5 \%$ para todos os órgãos. Os valores obtidos nesse estudo foram sistematicamente menores que os valores de Hadid et al. [9]. Os maiores desvios foram observados para ovário $(-3,8 \%)$, e parede da vesícula biliar (-3,6\%). Mucosa oral e estomago apresentaram os menores desvios $(-0,5 \%)$ e $(-0,7 \%)$ respectivamente. As diferenças entre as doses efetivas foram menores que 2,5\% para RCP_AF.

A média do desvio dos valores obtidos nesse trabalho em relação aos valores de referência de Zankl et al. [8] foi de -0,3\% para RCP_AM e -0,4\% para RCP_AF. Considerando os VR de Hadid et al. [9], a média dos desvios foi $-2,9 \%$ e $-2,2 \%$ para RCP_AM e RCP_AF, respectivamente. Tal fato confirma a tendência de subestimativa previamente mencionada.

A comparação da média dos desvios entre os VR demonstra que Zankl et al. [8], também obteve valores sistematicamente menores que os valores de Hadid, et al. [9]: -2,6\% para RCP_AM e -1,8\% para RCP_AF. Os órgãos que apresentaram maiores discrepâncias foram músculo cardíaco (-7,2\%), timo $(-6,3 \%)$ e parede da vesícula biliar $(-5,9 \%)$ para RCP_AM. No caso de RCP_AF as maiores diferenças foram encontradas no baço $(-3,7 \%)$, pâncreas $(-3,5 \%)$ e medula óssea vermelha $(-3,3 \%)$.

\section{DISCUSSÃO}

Os valores de dose média absorvida nos órgãos por unidade de atividade injetada calculados por meio dos protocolos de dosimetria interna desenvolvidos por nosso grupo não foram significativamente diferentes dos valores de referência. Nos testes estatísticos foram considerados 
erros relativos de $5 \%$ para os valores de referência e nível de significância 0,01 . Nossos resultados ainda permanecem estatisticamente iguais aos de Zankl et al. [8], considerando todos os órgãos dos modelos, para erros relativos acima de 1,5\% para RCP_AM e de 1,5\% para RCP_AF, mantendo o nível de significância $(0,01)$. Tais valores seriam 2,8\% (RCP_AM) e 1,3\% (RCP_AF) considerando os VR de Hadid et al. [9].

No trabalho de Zankl et al. [8], as Frações Específicas Absorvidas (SAF) de fótons e elétrons foram geradas por códigos de Monte Carlo distintos. EGSnrc foi utilizado nas simulações de transporte de elétrons [8]. O transporte de fótons foi simulado separadamente [8]. Um programa desenvolvido na instituição foi utilizado para calcular os coeficientes de dose absorvida por atividade injetada para os órgãos de interesse a partir dos SAF de fótons e elétrons previamente obtidos. Essa metodologia é ligeiramente distinta da metodologia utilizada por nosso grupo. Nesse estudo apenas um arquivo de entrada para o MCNPx foi gerado para cada Fantoma. Pósitrons foram simulados com biodistribuição da ${ }^{18}$ F-FDG baseada nos dados da ICRP 128 [12]. Tal procedimento assemelha-se mais com o utilizado por Hadid et al. [9]. Contudo, nossos resultados correlacionam-se melhor com os de Zankl, et al. [8].

Uma das diferenças entre a simulação de pósitrons e a de elétrons/fótons correspondentes é que no primeiro caso, uma fração pósitrons emitidos escapa do órgão-fonte e a aniquilação, emissão dos fótons de $511 \mathrm{keV}$, ocorre fora do órgão. Assim, no caso de pósitrons, o órgão-fonte pode apresentar dose absorvida menor e os órgãos adjacentes tendem a apresentar um acréscimo na dose quando comparados com a utilização de fontes elétron/fóton. Tal efeito é mais pronunciado para pósitrons com maior energia e em órgãos pequenos e/ou como maior relação superfície/volume. Tireoide, glândulas suprarrenais, baço, pâncreas, esôfago, parede de órgãos (coração, intestinos, estomago, v. biliar, bexiga) e muitos ossos são exemplos de órgãos que se encaixam nessas condições. Apesar disso, estudos ainda não publicados por nosso grupo demonstraram que simulações com ${ }^{18}$ F-FDG, utilizando os fantomas RCP_AM e RCP_AF, não apresentaram diferenças estatisticamente significativas entre a utilização de fontes de pósitrons e de elétrons/fótons. Além disso, testes prévios com esses modelos demostraram que os tallys $+\mathrm{F} 6$ e *F8 produzem resultados idênticos quando "MODE p e" é utilizado, mesmo para órgãos pequenos ou irregulares. 
Os valores apresentados por Hadid et al. [9] foram sistematicamente maiores que os valores obtidos nesse trabalho e os calculados por Zankl et al. [8]. Esses desvios, apesar de não serem estatisticamente significativos, podem indicar pequenas divergências na metodologia de cálculo. Os desvios também podem ter ocorrido devido a diferença entre as versões do MCNPx utilizadas em nosso estudo (2.7.0) e no de Hadid et al. [9] (2.6f).

\section{CONCLUSÕES}

Protocolos de dosimetria interna (PDI) foram desenvolvidos, baseados nos fantomas adulto feminino e masculino voxelizados de referência, rotinas computacionais in house, e código estocástico de transporte de partículas MCNPx. Considerando a validação gerada para a estimativa da dose efetiva produzida pela aplicação de ${ }^{18} \mathrm{~F}-\mathrm{FDG}$, os protocolos de dosimetria interna desenvolvidos por nosso grupo apresentam dados dosimétricos considerados satisfatórios visto que não foram estatisticamente diferentes dos valores de referência [8, 9].

Os dados dosimétricos baseados nos PDI desenvolvidos correlacionaram-se melhor com os de Zankl, et al. [8]. Os valores de Hadid et al. [9], apesar de estatisticamente iguais aos gerados nesse trabalho, foram sistematicamente maiores (em média 2,6\%). A determinação de quão significativa é essa tendência e sua origem demandaria uma discriminação aprofundada na metodologia empregada nos trabalhos de referência.

Programas e rotinas foram desenvolvidos in house para possibilitar a elaboração dos PID descritos nesse trabalho. O conjunto desses desenvolvimentos possibilitará trabalhos mais específicos, direcionados à dosimetria personalizada.

Acreditamos que um maior detalhamento da metodologia de cálculo utilizada, juntamente com a apresentação do desvio padrão da dose absorvida média de cada órgão, facilitará a realização futura de estudos comparativos por outros pesquisadores. 


\section{AGRADECIMENTOS}

Gostaríamos de agradecer ao Laboratório de Metrologia de Nêutrons do Instituto de Radioproteção e Dosimetria (IRD/CNEN) por permitir o acesso e uso do cluster de alta-performance para realização das simulações em MCNPx. Esse projeto de pesquisa foi apoiado pelas seguintes instituições brasileiras: Fundação de Amparo à Pesquisa de Minas Gerais (FAPEMIG) e Conselho Nacional de Desenvolvimento Científico e Tecnológico (CNPq - Edital REBRAT/SUS).

\section{REFERENCES}

1. ICRP Publication 110. Adult Reference Computational Phantoms. Ann ICRP, v. 39(2), p. 1-166, 2009.

2. ICRP Publication 103. The 2007 Recommendations of the International Commission on Radiological Protection. Ann ICRP, v. 37(2-4), p. 1-332, 2007.

3. ICRP Publication 133. The ICRP Computational Framework for Internal Dose Assessment for Reference Adults: Specific Absorbed Fractions. Ann. ICRP, v.45(2), p.1-73, 2016.

4. TRINDADE, B. M.; CAMPOS, T. P. R. Sistema computacional para dosimetria de nêutrons e fótons baseado em métodos estocásticos aplicado a radioterapia e radiologia. Radiol Bras, v. 44(2), p. 109-116, 2011.

5. MENDES, B. M.; ALMEIDA, I. G.; TRINDADE, B. M.; CAMPOS, T. P. R. A female voxel phantom for radiopharmaceuticals internal dosimetry [resumo]. In: $4^{\text {th }}$ International Nuclear Chemistry Congress; 2014, Maresias - SP - Brasil. $4^{\text {th }}$ INCC - Abstract Book, p. 1-345, 2014.

6. MENDES, B. M.; FONSECA, T. C. F.; ALMEIDA, I.G.; TRINDADE, B. M.; CAMPOS T. P. R. Validation of Internal Dosimetry Protocols based in stochastic method. In: International Nuclear Atlantic Conference - INAC 2015; 2015, São Paulo, SP, Brazil.

7. PAIVA, F. G.; OlIVEIRA, A. H.; MENDES, B. M.; PINTO, J. R.; FILHO, N. A. N.; DANTAS, B. M.; DANTAS, A. L. A.; SILVA, T.A.; LACERDA, M. A. S.; FONSECA, T. C. F. Improvement of the WBC calibration of the Internal Dosimetry Laboratory of the CDTN/CNEN using the physical phantom BOMAB and MCNPx Code. Appl Radiat Isot, v.117, p. 26-30, 2015.

8. ZANKL, M.; SCHLATTL, H.; PETOUSSI-HENSS, N.; HOESCHEN, C. Electron specific absorbed fractions for the adult male and female ICRP/ICRU reference computational phantoms.

Phys Med Biol, v. 57, p. 4501-4526, 2012. 
9. HADID, L.; GARDUMI, A.; DESBRÉE, A. Evaluation of absorbed and effective doses to patients from radiopharmaceuticals using the ICRP 110 reference computational phantoms and ICRP 103 formulation. Radiat Prot Dosimetry, v. 156(2), p. 141-159, 2013.

10. PELOWITZ, D. B., ed. MCNPx ${ }^{\text {TM }}$ User's Manual - Version 2.7.0. LA-CP-11-00438, 2011. 645p.

11. ICRP Publication 107. Nuclear Decay Data for Dosimetric Calculations. Ann ICRP, v. 38(3), p. 1-96, 2008.

12. ICRP Publication 128. Radiation Dose to Patients from Radiopharmaceuticals: a Compendium of Current Information Related to Frequently Used Substances. Ann ICRP, v. 44(2S), p.1-315, 2015.

13. RAYNAERT, N.; PALMANS, H.; THIERENS, H.; JERAJ, R. Parameter dependence of the MCNP electron transport in determining dose distributions. Med Phys, v. 29(10), p. 2446-2454, 2002. 